\title{
A Medication Distribution Model against Ebola
}

\author{
Peigen Zou \\ School of North China Electric Power University, Baoding, China \\ 1739687126@qq.com
}

Keywords: SIR model; medication distribution; AHP.

\begin{abstract}
The epidemic of Ebola which has stirred up worldwide concern calls for efforts to control the infection. We present an infection spread model to visualize the tendency of Ebola epidemic and devise a medication distribution model embedding Analytic Hierarchy Process to eradicate the spread of Ebola. Firstly, we choose Guinea, Sierra Leone and Liberia as target countries. Then the SIR (Susceptible, Infective, and Removal) model is developed to analyze the spread of Ebola and predict the possible future patients in the three countries. Thus we are able to calculate the total required amount of medication. Next, we adapt the Analytic Hierarchy Process (AHP) in our medication distribution model. We build an index set to evaluate the severity of countries so as to determine the exact amount of medication delivered to each country.
\end{abstract}

\section{Introduction.}

The ongoing Ebola outbreak in West Africa has drawn attention to public health worldwide. Within less than a year after its epidemic started in December 2013 in Guinea, Ebola virus (EBOV) has spread over a number of West-African countries (Guinea, Sierra Leone and Liberia) with high fatality rates up to $90 \%$ in humans. Fortunately, the World Medical Association has announced that their new medication could stop Ebola and cure patients whose disease is not advanced. This major progress presents possibilities of eradicating the Ebola virus,.In order to make the best use of the newly-invented medication, we need to make reasonable arrangement to guarantee that the distribution of the medication could meet the demands of the patients in different areas.

\section{Ebola spread model}

We find the SIR model that can be used in exploring the spread of Ebola since the SIR model has a particular application in a wide range of infections [1].The ordinary differential equations of the SIR model can be presented as

$$
\left\{\begin{array}{l}
\frac{d I}{d t}=\lambda S I-\mu I \\
\frac{d S}{d t}=-\lambda S I \\
\frac{d R}{d t}=\mu I
\end{array}\right.
$$

$S$ refers to the number of the susceptible people, $I$ refers to the number of the infectious people and $R$ refers to the number of the people recovered, $\lambda$ refers to the daily contact rate, $\mu$ refers to daily cure rate[2-3].

We choose Guinea, Sierra Leone and Liberia as target countries. As now we grasp the principle of the SIR model, a prediction of the future infectious people can be obtained according to the collected data .By selecting the suitable value of $\lambda$ and $\mu$, we can calculate the maximum number of the percentage of the patients in each country. The result can be listed as follows: 
Table 1. The calculation of SIR model

\begin{tabular}{ccccc}
\hline & $\lambda$ & $\mu$ & $I_{\max }$ & Population \\
\hline Guinea & 0.0185 & 0.003 & 0.54296 & 11.2 million \\
Liberia & 0.0320 & 0.003 & 0.68443 & 4.3 million \\
Sierra Leone & 0.0335 & 0.006 & 0.51297 & 6.1 million \\
\hline
\end{tabular}

The maximum infectious patients can be calculated by

$I^{*}=\sum I_{\max } \cdot$ Populalion

$I^{*}$ refers to the maximum number of infectious people.

The required amount of medication can be estimated based on the number of infectious people.

\section{Medication distribution model}

In view of the evaluation of the various demands for the medication in different countries, we adapt the analytic hierarchy process (AHP) to tackle the problem. The analytic hierarchy process (AHP) is a structured technique for organizing and analyzing complex decisions, based on mathematics and psychology. It provides a comprehensive and rational framework for structuring a decision problem, for representing and quantifying its elements, for relating those elements to overall goals, and for evaluating alternative solutions [4].

Next, we will crystallize each hierarchy of AHP.The first hierarchy, namely the overall goal, contains the requisite amount of the medication of each country. The second hierarchy, criterion hierarchy contains the index we mainly consider during the evaluation process. We construct three main index listed as follows:the proportion of morbidity ,mortality of Ebola in each country,the level of economic development of each country.The third hierarchy contains the three countries we select to work on.

Then we can obtain the matrix of the overall goal hierarchy:

$$
A=\left[\begin{array}{ccc}
1 & 1 / 2 & 4 \\
2 & 1 & 5 \\
1 / 4 & 1 / 5 & 1
\end{array}\right]
$$

The solution of the first matrix allowed us to solve the second matrix. From the matrix we conclude the maximum eigen value $\lambda_{\max }=3.0246$, and the relevant eigen vector is:

$$
W_{0}=\left(\begin{array}{lll}
0.3331 & 0.5695 & 0.0974
\end{array}\right)^{T}
$$

This process requires data standardization and mapping the data to the scale $1 \sim 9$. Next, we will take the consistency test upon the judgment matrix.We define the consistence index as:

$$
C I=\frac{\lambda_{\max }-n}{n-1}
$$

In this premise, we generate the matrix of 500 samples using the stochastic method: extract numbers and their reciprocals from scale 1 9 randomly to build the judgment matrix. We figure out the max Eigen value of the matrix and take the average.

Table 1. The value of RI

\begin{tabular}{cccccccccc}
\hline $\mathrm{n}$ & 1 & 2 & 3 & 4 & 5 & 6 & 7 & 8 & 9 \\
\hline $\mathrm{RI}$ & 0 & 0 & 0.58 & 0.90 & 1.12 & 1.24 & 1.32 & 1.41 & 1.45 \\
\hline
\end{tabular}

We define the equation as:

$$
R I=\frac{\lambda_{\max }{ }^{\prime}-n}{n-1}
$$

Accordingly, the consistency ratio can be expressed as: 


$$
C R=\frac{C I}{R I}
$$

As is shown in the calculation: $C R=0.0212<0.1$, we believe that the consistency test of the matrix is acceptable. After all the matrixes passing the consistence test, we can calculate the index weight according to the matrix. We now ensure that all the matrixes are consistent matrixes, then we can use maximum Eigen vector to represent to what extent the former hierarchy influence the next one.

$$
W_{0}=\left(\begin{array}{lll}
0.3331 & 0.5695 & 0.0974
\end{array}\right)^{T}
$$

We calculate the weight between the hierarchies. Finally, we get the weight matrix $W$ according to the 3 elements from the criterion hierarchy.

$$
W=\left(\begin{array}{lll}
w_{1} & w_{2} & w_{3}
\end{array}\right)^{T}=\left[\begin{array}{lll}
0.1322 & 0.4723 & 0.3155 \\
0.3909 & 0.3084 & 0.4061 \\
0.4769 & 0.2194 & 0.2783
\end{array}\right]
$$

We can get any ranking weight of the three countries by solving the equation below:

$$
c_{i}=\sum_{j=1}^{3} W_{i j} W_{0 j}
$$

By calculating the matrix below we can get the weight of the quantity of medication in the selected countries.

$$
c=W \cdot W_{0}=\left(\begin{array}{lll}
0.3438 & 0.3454 & 0.3109
\end{array}\right)^{T}
$$
figure.

The medication distribution ratio of the three countries can be observed visually in the following

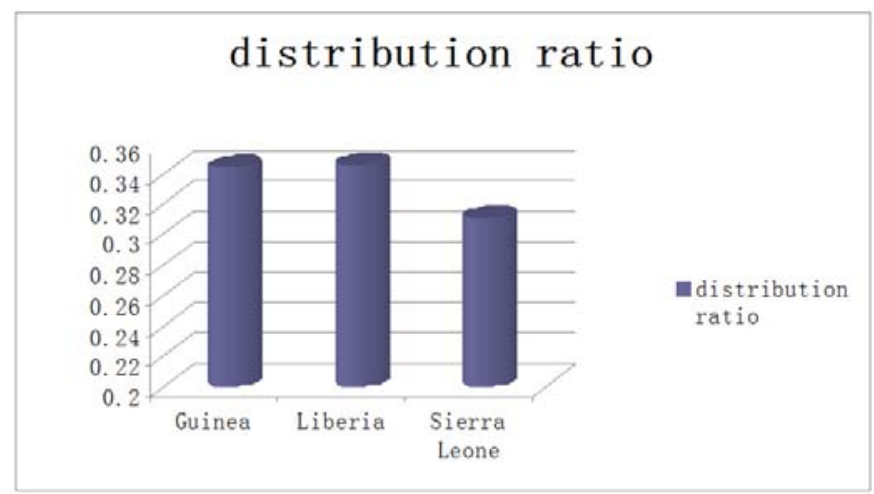

Figure 1. The distribution ratio of Guinea, Liberia and Sierra Leone

\section{Conclusion}

We consider synthetically of the main index of each country, including the percentage of patients, mortality and the level of economic development. We come to a reasonable distribution scheme of the medication.

\section{References}

[1] Bai Fu-mei, Infectious disease model [J]. Journal of Taiyuan Normal University (Natural Science Edition ), 2012, 11:53:56.

[2] Zhou Hou-qing,Xu You-zhuan. A mathematical model on the quantity of people infected by ebola virus. [J]. Journal of Shaoyang University( Natural Science Edition), 2014,04:1-5.

[3] Wang Ke-chuang, Liu Xiang. Study of SARS epidemic model [J]. Science \& Technology Information, 2008,16:42-43.

[4] Guo Jin-yu, Zhang Zhong-bin, Sun Qing-yun. The study and applications of analytic hierarchy process [J]. China Safety Science Journal, 2008,05:148-153. 